\title{
3-P-350 Poster Sessions
}

\section{Establishment and characterization of a novel murine model for head and neck cancer cachexia}

\section{Zi-Xuan Hong ${ }^{1}$, Kuo-Chu Lai ${ }^{2}$}

${ }^{I}$ Ms program in Pharmacol. \& Toxicol., Dept. Med., Sch. Med., Tzu Chi Univ., Hualien, Taiwan, ${ }^{2}$ Dept. Pharmacol., Sch. Med., Tzu Chi Univ., Hualien, Taiwan

Cancer cachexia is the metabolic wasting syndrome that the cancer will release a lot of cytokines and result in metabolic abnormalities and anorexia. The rate of the cancer death has been revealed almost $30 \%$ in the cachexia patients. Cancer cachexia can vary according to tumor type, site, mass, and host genotype. Clinical studies showed that more than $60 \%$ of head and neck cancer (HNC) patients might develop cancer cachexia. Several animal models have been established to elucidate the importance of pro-cachectic cytokines, such as TNF- $\alpha$ and interleukin 6 (IL-6) in the pathogenesis of cancer cachexia. Unfortunately, the pathogenesis of HNC cachexia is still unknown. Our preliminary results demonstrated that IFIT2, an interferon-induced protein with tetratricopeptide repeat 2 (IFIT2) depletion enhances expression of TNF- $\alpha$, a well-known cancer-cachexia related cytokine in HNC cells. Thus, this study aims to explore the effect of IFIT2 depletion on HNC cachexia. To the end, a murine model was established by injecting the IFIT2 depleted HNC cells. Moreover, the body weight and survival rate were significantly decreased in IFIT2-depleted cells bearing mice as compared to control mice. The quadriceps had a $28.6 \%$ reduction in cachectic mice. Similarly, the gastrocnemius had a $33.3 \%$ reduction in cachectic mice. These results suggest that IFIT2depleted HNC cells bearing mice may act as a model for studies on HNC cachexia. 